\title{
An Opial-Type Inequality on Time Scales
}

\author{
Qiao-Luan $\mathrm{Li}^{1}$ and Wing-Sum Cheung ${ }^{2}$ \\ ${ }^{1}$ College of Mathematics and Information Science, Hebei Normal University, Shijiazhuang 050024, China \\ ${ }^{2}$ Department of Mathematics, The University of Hong Kong, Hong Kong
}

Correspondence should be addressed to Qiao-Luan Li; q1171125@163.com

Received 4 January 2013; Accepted 16 January 2013

Academic Editor: Allan Peterson

Copyright (C) 2013 Q.-L. Li and W.-S. Cheung. This is an open access article distributed under the Creative Commons Attribution License, which permits unrestricted use, distribution, and reproduction in any medium, provided the original work is properly cited.

We establish some new Opial-type inequalities involving higher order delta derivatives on time scales. These extend some known results in the continuous case in the literature and provide new estimates in the setting of time scales.

\section{Introduction}

Opial's inequality appeared for the first time in 1960 in [1] and has been receiving continual attention throughout the years (cf., e.g., [2-7]). The inequality together with its numerous generalizations, extensions, and discretizations has been playing a fundamental role in the study of the existence and uniqueness properties of solutions of initial and boundary value problems for differential equations as well as difference equations $[8,9]$. Two excellent surveys on these inequalities can be found in $[10,11]$.

In 1960, Opial established the following integral inequality.

Theorem A (see [1]). If $f \in C^{1}[0, h]$ satisfies $f(0)=f(h)=0$ and $f(x)>0$ for all $x \in(0, h)$, then

$$
\int_{0}^{h}\left|f(x) f^{\prime}(x)\right| d x \leq \frac{h}{4} \int_{0}^{h}\left|f^{\prime}(x)\right|^{2} d x
$$

Shortly after the publication of Opial's paper, Olech provided a modified version of Theorem A. His result is stated in the following.

Theorem B (see [12]). If $f$ is absolutely continuous on $[0, h]$ with $f(0)=0$, then

$$
\int_{0}^{h}\left|f(x) f^{\prime}(x)\right| d x \leq \frac{h}{2} \int_{0}^{h} f^{\prime}(x)^{2} d x .
$$

The equality in (2) holds if and only if $f(x)=c x$, where $c$ is a constant.

The first natural extension of Opial's inequality (1) involving higher order derivatives $x^{(n)}(s)(n \geq 1)$ is embodied in the following.

Theorem C (see [10]). Let $x(t) \in C^{(n)}[0, a]$ be such that $x^{(i)}(0)=0,0 \leq i \leq n-1(n \geq 1)$. Then the following inequality holds:

$$
\int_{0}^{a}\left|x(t) x^{(n)}(t)\right| d t \leq \frac{1}{2} a^{n} \int_{0}^{a}\left|x^{(n)}(t)\right|^{2} d t .
$$

In 1997, Alzer [13] considered Opial-type inequalities which involve higher-order derivatives of two functions. These generalize earlier results of Agarwal and Pang [14].

In this paper, we consider the Opial-type inequality which involves higher-order delta derivatives of two functions on time scales. Our results in special cases yield some of the recent results on Opial's inequality and provide some new estimates on such types of inequalities in this general setting.

\section{Main Results}

Let $\mathbb{T}$ be a time scale; that is, $\mathbb{T}$ is an arbitrary nonempty closed subset of real numbers. Let $a, b \in \mathbb{T}$. We suppose that the reader is familiar with the basic features of calculus on time scales for dynamic equations. Otherwise one can consult 
Bohner and Peterson's book [15] for most of the materials needed.

We first quote the following elementary lemma and the delta time scales Taylor formula.

Lemma 1 (see [16]). Let $a \geq 0, p \geq 1$ be real constants. Then

$$
a^{1 / p} \leq \frac{1}{p} k^{(1-p) / p} a+\frac{p-1}{p} k^{1 / p}
$$

for any $k>0$.

Lemma 2 (see [17]). Let $f \in C_{r d}^{m}(\mathbb{\mathbb { T }})=$ the set of functions that are $m$ times differentiable with $r d$-continuous derivatives on $\mathbb{T}, m \in \mathbb{N}$. Then for any $a, b \in \mathbb{T}$ and $t \in[a, b] \cap \mathbb{T}$,

$$
\begin{aligned}
f(t)= & \sum_{k=0}^{m-1} h_{k}(t, a) f^{\Delta^{k}}(a) \\
& +\int_{a}^{t} h_{m-1}(t, \sigma(\tau)) f^{\Delta^{m}}(\tau) \Delta \tau,
\end{aligned}
$$

where $h_{0}(t, s):=1, h_{n+1}(t, s):=\int_{s}^{t} h_{n}(\tau, s) \Delta \tau, n \in \mathbb{N}$.

Our main results are given in the following theorems.

Theorem 3. Let $0 \leq r \leq s<t, s>0, t>1$ be real numbers, and let $m, k$ be integers with $0 \leq k \leq m-1$. Let $p>0$ and $q \geq 0$ be measurable functions on $\Upsilon:=[a, b] \cap \mathbb{T}$. Further, let $f, g \in$ $C_{r d}^{m-1}(\Upsilon)$ with $f^{\Delta^{i}}(a)=g^{\Delta^{i}}(a)=0, i=0,1, \ldots, m-1$, and let $f^{\Delta^{m-1}}, g^{\Delta^{m-1}}$ be absolutely continuous on $\Upsilon$ such that the integrals $\int_{a}^{b} p(x)\left|f^{\Delta^{m}}(x)\right|^{t} \Delta x$ and $\int_{a}^{b} p(x)\left|g^{\Delta^{m}}(x)\right|^{t} \Delta x$ exist. Then one has

$$
\begin{aligned}
\int_{a}^{b} q(x) & {\left[\left|f^{\Delta^{k}}(x)\right|^{r}\left|g^{\Delta^{m}}(x)\right|^{s}\right.} \\
& \left.+\left|g^{\Delta^{k}}(x)\right|^{r}\left|f^{\Delta^{m}}(x)\right|^{s}\right] \Delta x \\
\leq & 2^{1-\alpha}\left(\int_{a}^{b} h(x)^{t /(t-s)} \Delta x\right)^{1-\alpha} \\
& \cdot\left[\beta k^{\beta-1} F(b) G(b)+(1-\beta) k^{\beta}(F(b)+G(b))\right]^{\alpha},
\end{aligned}
$$

$$
\begin{aligned}
& F(x):=\int_{a}^{x} p(\tau)\left|f^{\Delta^{m}}(\tau)\right|^{t} \Delta \tau, \\
& G(x):=\int_{a}^{x} p(\tau)\left|g^{\Delta^{m}}(\tau)\right|^{t} \Delta \tau .
\end{aligned}
$$

Proof. Since $f^{\Delta^{i}}(a)=0, i=0,1, \ldots, m-1$, we obtain from Taylor's theorem that for all $x \in \Upsilon$,

$$
f(x)=\int_{a}^{x} h_{m-1}(x, \sigma(\tau)) f^{\Delta^{m}}(\tau) \Delta \tau,
$$

and hence

$$
f^{\Delta^{k}}(x)=\int_{a}^{x} h_{m-k-1}(x, \sigma(\tau)) f^{\Delta^{m}}(\tau) \Delta \tau .
$$

From (9) and Hölder's inequality we get

$$
\begin{aligned}
\left|f^{\Delta^{k}}(x)\right| \leq & \int_{a}^{x} h_{m-k-1}(x, \sigma(\tau))\left|f^{\Delta^{m}}(\tau)\right| \Delta \tau \\
= & \int_{a}^{x} h_{m-k-1}(x, \sigma(\tau)) p(\tau)^{-1 / t} p(\tau)^{1 / t}\left|f^{\Delta^{m}}(\tau)\right| \Delta \tau \\
\leq & {\left[\int_{a}^{x} h_{m-k-1}^{t /(t-1)}(x, \sigma(\tau)) p(\tau)^{1 /(1-t)} \Delta \tau\right]^{1-1 / t} } \\
& \cdot\left(\int_{a}^{x} p(\tau)\left|f^{\Delta^{m}}(\tau)\right|^{t} \Delta \tau\right)^{1 / t} \\
= & P(x)^{1-1 / t} F(x)^{1 / t},
\end{aligned}
$$

where $P(x):=\int_{a}^{x} h_{m-k-1}^{t /(t-1)}(x, \sigma(\tau)) p(\tau)^{1 /(1-t)} \Delta \tau, \quad F(x):=$ $\int_{a}^{x} p(\tau)\left|f^{\Delta^{m}}(\tau)\right|^{t} \Delta \tau$.

Let

$$
G(x):=\int_{a}^{x} p(\tau)\left|g^{\Delta^{m}}(\tau)\right|^{t} \Delta \tau
$$

Then we have

$$
\left|g^{\Delta^{m}}(x)\right|^{s}=G^{\Delta}(x)^{s / t} p(x)^{-s / t} .
$$

So (10) together with (12) implies

$$
q(x)\left|f^{\Delta^{k}}(x)\right|^{r}\left|g^{\Delta^{m}}(x)\right|^{s} \leq h(x) F(x)^{r / t} G^{\Delta}(x)^{s / t},
$$

where $h(x):=q(x) p(x)^{-s / t} P(x)^{r(t-1) / t}$. Integrating both sides of (13) over $\Upsilon$ and making use of Hölder's inequality, we obtain

$$
\begin{aligned}
\int_{a}^{b} q(x)\left|f^{\Delta^{k}}(x)\right|^{r}\left|g^{\Delta^{m}}(x)\right|^{s} \Delta x \\
\quad \leq \int_{a}^{b} h(x) F(x)^{r / t} G^{\Delta}(x)^{s / t} \Delta x \\
\quad \leq\left(\int_{a}^{b} h(x)^{t /(t-s)} \Delta x\right)^{1-s / t}\left(\int_{a}^{b} F(x)^{r / s} G^{\Delta}(x) \Delta x\right)^{s / t} .
\end{aligned}
$$

Similarly, we get

$$
\begin{aligned}
\int_{a}^{b} q(x)\left|g^{\Delta^{k}}(x)\right|^{r}\left|f^{\Delta^{m}}(x)\right|^{s} \Delta x & \\
& \leq\left(\int_{a}^{b} h(x)^{t /(t-s)} \Delta x\right)^{1-s / t} \cdot\left(\int_{a}^{b} G(x)^{r / s} F^{\Delta}(x) \Delta x\right)^{s / t} .
\end{aligned}
$$


Abstract and Applied Analysis

3

Recall the elementary inequalities

$$
c_{\alpha}(A+B)^{\alpha} \leq A^{\alpha}+B^{\alpha} \leq d_{\alpha}(A+B)^{\alpha}, \quad(A, B \geq 0),
$$

where

$$
\begin{aligned}
c_{\alpha} & := \begin{cases}1, & 0 \leq \alpha \leq 1, \\
2^{1-\alpha}, & \alpha \geq 1,\end{cases} \\
d_{\alpha} & := \begin{cases}2^{1-\alpha}, & 0 \leq \alpha \leq 1, \\
1, & \alpha \geq 1 .\end{cases}
\end{aligned}
$$

Let $\beta=r / s$. Since $\alpha=s / t \in(0,1)$ and $F$ is nondecreasing, from (14)-(16), we have

$$
\begin{aligned}
& \int_{a}^{b} q(x)\left[\left|f^{\Delta^{k}}(x)\right|^{r}\left|g^{\Delta^{m}}(x)\right|^{s}+\left|g^{\Delta^{k}}(x)\right|^{r}\left|f^{\Delta^{m}}(x)\right|^{s}\right] \Delta x \\
& \leq\left(\int_{a}^{b} h(x)^{t /(t-s)} \Delta x\right)^{1-\alpha} \\
& \cdot\left[\left(\int_{a}^{b} F^{\Delta}(x) G^{\beta}(x) \Delta x\right)^{\alpha}+\left(\int_{a}^{b} G^{\Delta}(x) F^{\beta}(x) \Delta x\right)^{\alpha}\right] \\
& \leq\left(\int_{a}^{b} h(x)^{t /(t-s)} \Delta x\right)^{1-\alpha} \\
& \cdot 2^{1-\alpha}\left(\int_{a}^{b}\left[F^{\Delta}(x) G^{\beta}(x)+G^{\Delta}(x) F^{\beta}(x)\right] \Delta x\right)^{\alpha} \\
& \leq\left(\int_{a}^{b} h(x)^{t /(t-s)} \Delta x\right)^{1-\alpha} \\
& \cdot 2^{1-\alpha}\left(\int_{a}^{b}\left[F^{\Delta}(x) G^{\beta}(x)+G^{\Delta}(x) F^{\beta}(\sigma(x))\right] \Delta x\right)^{\alpha} .
\end{aligned}
$$

By Lemma 1, we get

$$
\begin{aligned}
\int_{a}^{b}[ & \left.F^{\Delta}(x) G^{\beta}(x)+G^{\Delta}(x) F^{\beta}(\sigma(x))\right] \Delta x \\
\leq & \int_{a}^{b}\left[\beta k^{\beta-1} G(x) F^{\Delta}(x)+(1-\beta) k^{\beta} F^{\Delta}(x)\right. \\
& \left.\quad+\beta k^{\beta-1} F(\sigma(x)) G^{\Delta}(x)+(1-\beta) k^{\beta} G^{\Delta}(x)\right] \Delta x \\
= & \beta k^{\beta-1} \int_{a}^{b}\left[G(x) F^{\Delta}(x)+F(\sigma(x)) G^{\Delta}(x)\right] \Delta x \\
& +(1-\beta) k^{\beta}\left[\int_{a}^{b} F^{\Delta}(x) \Delta x+\int_{a}^{b} G^{\Delta}(x) \Delta x\right] \\
= & \beta k^{\beta-1} F(b) G(b)+(1-\beta) k^{\beta}[F(b)+G(b)] .
\end{aligned}
$$

(19)
From (18) and (19), we conclude

$$
\begin{gathered}
\int_{a}^{b} q(x)\left[\left|f^{\Delta^{k}}(x)\right|^{r}\left|g^{\Delta^{m}}(x)\right|^{s}+\left|g^{\Delta^{k}}(x)\right|^{r}\left|f^{\Delta^{m}}(x)\right|^{s}\right] \Delta x \\
\leq 2^{1-\alpha}\left(\int_{a}^{b} h(x)^{t /(t-s)} \Delta x\right)^{1-\alpha} \\
\cdot\left[\beta k^{\beta-1} F(b) G(b)+(1-\beta) k^{\beta}(F(b)+G(b))\right]^{\alpha} .
\end{gathered}
$$

The proof is complete.

Theorem 4. Let $r \geq 0, s>0, s<t, t>1$ be real numbers, and let $m, k$ be integers with $0 \leq k \leq m-1$. Let $p>0$, and $q \geq 0$ be measurable functions on $\Upsilon:=[a, b] \cap \mathbb{T}$. Further, let $f, g \in$ $C_{r d}^{m-1}(\Upsilon)$ with let $f^{\Delta^{i}}(a)=g^{\Delta^{i}}(a)=0, i=0,1, \ldots, m-1$, and $f^{\Delta^{m-1}}, g^{\Delta^{m-1}}$ be absolutely continuous on $\Upsilon$ such that the integrals $\int_{a}^{b} p(x)\left|f^{\Delta^{m}}(x)\right|^{t} \Delta x$ and $\int_{a}^{b} p(x)\left|g^{\Delta^{m}}(x)\right|^{t} \Delta x$ exist. Then one has

$$
\begin{gathered}
\int_{a}^{b} q(x)\left[\left|f^{\Delta^{k}}(x)\right|^{r}\left|g^{\Delta^{m}}(x)\right|^{s}+\left|g^{\Delta^{k}}(x)\right|^{r}\left|f^{\Delta^{m}}(x)\right|^{s}\right] \Delta x \\
\leq 2^{1-\alpha}\left(\int_{a}^{b} h(x)^{t /(t-s)} \Delta x\right)^{1-\alpha} \\
\cdot\left[d_{\beta} \Gamma(G+F)-\Gamma(G)-\Gamma(F)\right]^{\alpha},
\end{gathered}
$$

where $\Gamma(H):=\int_{a}^{b} H^{\beta} \Delta H, \beta:=r / s, \alpha:=s / t, h(x):=q(x) p(x)^{-\alpha}$ $P(x)^{r(t-1) / t}, P(x):=\int_{a}^{x} h_{m-k-1}^{t /(t-1)}(x, \sigma(\tau)) p(\tau)^{1 /(1-t)} \Delta \tau$, and

$$
d_{\beta}:= \begin{cases}2^{1-\beta}, & 0 \leq \beta \leq 1, \\ 1, & \beta \geq 1\end{cases}
$$

Proof. Following the proof of Theorem 3, we obtain

$$
\begin{gathered}
\int_{a}^{b} q(x)\left[\left|f^{\Delta^{k}}(x)\right|^{r}\left|g^{\Delta^{m}}(x)\right|^{s}+\left|g^{\Delta^{k}}(x)\right|^{r}\left|f^{\Delta^{m}}(x)\right|^{s}\right] \Delta x \\
\leq\left(\int_{a}^{b} h(x)^{t /(t-s)} \Delta x\right)^{1-\alpha} \\
\cdot 2^{1-\alpha}\left(\int_{a}^{b}\left[F^{\Delta}(x) G^{\beta} x+G^{\Delta}(x) F^{\beta}(x)\right] \Delta x\right)^{\alpha} .
\end{gathered}
$$


Using (16),

$$
\begin{aligned}
\int_{a}^{b} & {\left[F^{\Delta}(x) G^{\beta}(x)+G^{\Delta}(x) F^{\beta}(x)\right] \Delta x } \\
= & \int_{a}^{b}\left(G^{\beta}(x)+F^{\beta}(x)\right)\left(G^{\Delta}(x)+F^{\Delta}(x)\right) \Delta x \\
& \quad-\int_{a}^{b}\left(G^{\beta}(x) G^{\Delta}(x)+F^{\beta}(x) F^{\Delta}(x)\right) \Delta x \\
\leq & d_{\beta} \int_{a}^{b}(G(x)+F(x))^{\beta} \Delta(G(x)+F(x)) \\
& \quad-\int_{a}^{b} G^{\beta}(x) \Delta G(x)-\int_{a}^{b} F^{\beta}(x) \Delta F(x) \\
= & d_{\beta} \Gamma(G+F)-\Gamma(G)-\Gamma(F) .
\end{aligned}
$$

The proof is complete.

Remark 5. In the special case where $\mathbb{T}=\mathbb{R}$, Theorem 4 reduces to Theorem 1 of [13].

Theorem 6. Let $f \in C_{r d}^{m-1}(\Upsilon), \Upsilon:=[a, b] \cap \mathbb{T}$ be such that $f^{\Delta^{i}}(a)=0,0 \leq k \leq m-1$, let $f^{\Delta^{m-1}}(x)$ be absolutely continuous on $\Upsilon$, and let $\int_{a}^{b}\left|f^{\Delta^{m}}(x)\right|^{2} \Delta x<\infty$. Then

$$
\begin{gathered}
\int_{a}^{b}\left|f^{\Delta^{k}}(x) f^{\Delta^{m}}(x)\right| \Delta x \\
\leq\left(\int_{a}^{b} \int_{a}^{x} h_{m-k-1}^{2}(x, \sigma(\tau)) \Delta \tau \Delta x\right)^{1 / 2} \\
\cdot\left(\int_{a}^{b}\left|f^{\Delta^{m}}(\tau)\right|^{2} \Delta \tau\right)^{1 / 2} .
\end{gathered}
$$

Proof. From the hypotheses, we have

$$
\left|f^{\Delta^{k}}(x)\right| \leq \int_{a}^{x} h_{m-k-1}(x, \sigma(\tau))\left|f^{\Delta^{m}}(\tau)\right| \Delta \tau .
$$

Multiplying (26) by $\left|f^{\Delta^{m}}(x)\right|$ and using Cauchy-Schwarz inequality, we obtain

$$
\begin{aligned}
& \left|f^{\Delta^{k}}(x) f^{\Delta^{m}}(x)\right| \\
& \leq\left|f^{\Delta^{m}}(x)\right| \int_{a}^{x} h_{m-k-1}(x, \sigma(\tau))\left|f^{\Delta^{m}}(\tau)\right| \Delta \tau \\
& \leq\left|f^{\Delta^{m}}(x)\right|\left(\int_{a}^{x} h_{m-k-1}^{2}(x, \sigma(\tau)) \Delta \tau\right)^{1 / 2} \\
& \quad \cdot\left(\int_{a}^{x}\left|f^{\Delta^{m}}(\tau)\right|^{2} \Delta \tau\right)^{1 / 2} .
\end{aligned}
$$

Integrating both sides over $x$ from $a$ to $b$ and using Cauchy-Schwarz inequality, we observe

$$
\begin{aligned}
& \int_{a}^{b}\left|f^{\Delta^{k}}(x) f^{\Delta^{m}}(x)\right| \Delta x \\
& \leq {\left[\int_{a}^{b} \int_{a}^{x} h_{m-k-1}^{2}(x, \sigma(\tau)) \Delta \tau \Delta x\right]^{1 / 2} } \\
& \cdot\left[\int_{a}^{b}\left|f^{\Delta^{m}}(x)\right|^{2} \int_{a}^{x}\left|f^{\Delta^{m}}(\tau)\right|^{2} \Delta \tau \Delta x\right]^{1 / 2} \\
&= {\left[\int_{a}^{b} \int_{a}^{x} h_{m-k-1}^{2}(x, \sigma(\tau)) \Delta \tau \Delta x\right]^{1 / 2} } \\
& \cdot\left[\int_{a}^{b}\left|f^{\Delta^{m}}(\tau)\right|^{2} \Delta \tau\right]^{1 / 2} .
\end{aligned}
$$

The proof is complete.

Theorem 7. Let $p(x)>0, q(x)$ be nonnegative and measurable on $\Upsilon=[a, b] \cap \mathbb{T}$, and let $f \in C_{r d}^{m-1}(\Upsilon)$ be such that $f^{\Delta^{k}}(a)=0,0 \leq k \leq m-1$. If $f^{\Delta^{m-1}}(x)$ is absolutely continuous on $\Upsilon$, then for $r>1, r_{k}>0$, and any $0 \leq r_{m}<r$,

$$
\begin{aligned}
& \int_{a}^{b} q(x)\left|f^{\Delta^{m}}(x)\right|^{r_{m}}\left|f^{\Delta^{k}}(x)\right|^{r_{k}} \Delta x \\
& \leq\left[\int_{a}^{b} q(x)^{r /\left(r-r_{m}\right)} p(x)^{-r_{m} /\left(r-r_{m}\right)}\right. \\
& \left.\quad \times Q(x)^{(r-1) r_{m} /\left(r-r_{m}\right)} \Delta x\right]^{\left(r-r_{m}\right) / r} \Phi(y)^{r_{m} / r},
\end{aligned}
$$

where $Q(x):=\int_{a}^{x} h_{m-k-1}^{r / r-1}(x, \sigma(\tau)) p(\tau)^{-(r / r-1)} \Delta \tau, \Phi(y):=$ $\int_{a}^{b} y^{r_{k} / r_{m}} \Delta y(x), y(x):=\int_{a}^{x} p(\tau)\left|f^{\Delta^{m}}(\tau)\right|^{r} \Delta \tau$.

Proof. Following the hypotheses, it is easy to see that (26) holds. By using Hölder's inequality with indices $r$ and $r /(r-1)$, we obtain

$$
\begin{aligned}
\left|f^{\Delta^{k}}(x)\right| & \\
\leq & \int_{a}^{x} h_{m-k-1}(x, \sigma(\tau)) p(\tau)^{-1 / r} p(\tau)^{1 / r}\left|f^{\Delta^{m}}(\tau)\right| \Delta \tau \\
\leq & {\left[\int_{a}^{x} h_{m-k-1}^{r /(r-1)}(x, \sigma(\tau)) p(\tau)^{-r /(r-1)} \Delta \tau\right]^{(r-1) / r} } \\
& \cdot\left[\int_{a}^{x} p(\tau)\left|f^{\Delta^{m}}(\tau)\right|^{r} \Delta \tau\right]^{1 / r} \\
= & Q(x)^{(r-1) / r} y(x)^{1 / r},
\end{aligned}
$$

where $Q(x):=\int_{a}^{x} h_{m-k-1}^{r /(r-1)}(x, \sigma(\tau)) p(\tau)^{-r /(r-1)} \Delta \tau, y(x):=$ $\int_{a}^{x} p(\tau)\left|f^{\Delta^{m}}(\tau)\right|^{r} \Delta \tau$. So we get

$$
y^{\Delta}(x)=p(x)\left|f^{\Delta^{m}}(x)\right|^{r},
$$


and hence for any $r_{m}$,

$$
\left|f^{\Delta^{m}}(x)\right|^{r_{m}}=(p(x))^{-r_{m} / r}\left(y^{\Delta}(x)\right)^{r_{m} / r}
$$

Thus for $r_{k}>0$,

$$
\begin{gathered}
q(x)\left|f^{\Delta^{m}}(x)\right|^{r_{m}}\left|f^{\Delta^{k}}(x)\right|^{r_{k}} \\
\leq q(x)(p(x))^{-r_{m} / r}\left(y^{\Delta}(x)\right)^{r_{m} / r} \\
\times Q(x)^{(r-1) r_{k} / r} y(x)^{r_{k} / r} .
\end{gathered}
$$

Integrating both sides of (33) from $a$ to $b$ and applying Hölder's inequality with indices $r / r_{m}$ and $r /\left(r-r_{m}\right)$, we obtain

$$
\begin{aligned}
\int_{a}^{b} q(x)\left|f^{\Delta^{m}}(x)\right|^{r_{m}}\left|f^{\Delta^{k}}(x)\right|^{r_{k}} \Delta x \\
\leq \int_{a}^{b} q(x) p(x)^{-r_{m} / r}\left(y^{\Delta}(x)\right)^{r_{m} / r} Q(x)^{(r-1) r_{k} / r} y(x)^{r_{k} / r} \Delta x \\
\leq\left[\int_{a}^{b} q(x)^{r /\left(r-r_{m}\right)} p(x)^{-r_{m} /\left(r-r_{m}\right)} Q(x)^{(r-1) r_{k} /\left(r-r_{m}\right)} \Delta x\right]^{\left(r-r_{m}\right) / r} \\
\quad \cdot\left[\int_{a}^{b} y^{\Delta}(x) y(x)^{r_{k} / r_{m}} \Delta x\right]^{r_{m} / r} \\
=\left[\int_{a}^{b} q(x)^{r /\left(r-r_{m}\right)} p(x)^{-r_{m} /\left(r-r_{m}\right)}\right. \\
\left.\quad \times Q(x)^{(r-1) r_{k} /\left(r-r_{m}\right)} \Delta x\right]^{\left(r-r_{m}\right) / r} \Phi(y)^{r_{m} / r},
\end{aligned}
$$

where $\Phi(y):=\int_{a}^{b} y(x)^{r_{k} / r_{m}} \Delta y(x)$. The proof is complete.

Remark 8. In the special case where $\mathbb{T}=\mathbb{R}$, Theorems 6 and 7 reduce to Theorems 1 and 2 of [18].

\section{Acknowledgments}

The first author's research was supported by NSF of China (11071054), Natural Science Foundation of Hebei Province (A2011205012). The second author's research was partially supported by an HKU URG grant.

\section{References}

[1] Z. Opial, "Sur une inégalité," Annales Polonici Mathematici, vol. 8, pp. 29-32, 1960.

[2] G. A. Anastassiou, "Opial type inequalities involving RiemannLiouville fractional derivatives of two functions with applications," Mathematical and Computer Modelling, vol. 48, no. 3-4, pp. 344-374, 2008.

[3] W.-S. Cheung, Z. Dandan, and J. Pečarić, "Opial-type inequalities for differential operators," Nonlinear Analysis: Theory, Methods \& Applications, vol. 66, no. 9, pp. 2028-2039, 2007.
[4] W. S. Cheung, "Some generalized opial-type inequalities," Journal of Mathematical Analysis and Applications, vol. 162, no. 2, pp. 317-321, 1991.

[5] W. S. Cheung, "Opial-type inequalities with $m$ functions in $n$ variables," Mathematika, vol. 39, no. 2, pp. 319-326, 1992.

[6] C.-J. Zhao and W.-S. Cheung, "On some opial-type inequalities," Journal of Inequalities and Applications, vol. 2011, article 7, 2011.

[7] C. J. Zhao and W. S. Cheung, "On opial's type inequalities for an integral operator with homogeneous kernel," Journal of Inequalities and Applications, vol. 2012, article 123, 2012.

[8] R. P. Agarwal and V. Lakshmikantham, Uniqueness and Nonuniqueness Criteria for Ordinary Differential Equations, vol. 6 of Series in Real Analysis, World Scientific Publishing, Singapore, 1993.

[9] J. D. Li, "Opial-type integral inequalities involving several higher order derivatives," Journal of Mathematical Analysis and Applications, vol. 167, no. 1, pp. 98-110, 1992.

[10] R. P. Agarwal and P. Y. H. Pang, Opial Inequalities with Applications in Differential and Difference Equations, vol. 320 of Mathematics and Its Applications, Kluwer Academic, Dordrecht, The Netherlands, 1995.

[11] D. S. Mitrinović, J. E. Pečarić, and A. M. Fink, Inequalities Involving Functions and Their Integrals and Derivatives, vol. 53 of Mathematics and Its Applications (East European Series), Kluwer Academic, Dordrecht, The Netherlands, 1991.

[12] Z. Olech, "A simple proof of a certain result of Z. Opial," Annales Polonici Mathematici, vol. 8, pp. 61-63, 1960.

[13] H. Alzer, "An Opial-type inequality involving higher-order derivatives of two functions," Applied Mathematics Letters, vol. 10, no. 4, pp. 123-128, 1997.

[14] R. P. Agarwal and P. Y. H. Pang, "Sharp Opial-type inequalities involving higher order derivatives of two functions," Mathematische Nachrichten, vol. 174, pp. 5-20, 1995.

[15] M. Bohner and A. Peterson, Dynamic Equations on Time scales: An Introduction with Applications, Birkhäuser, Boston, Mass, USA, 2001.

[16] W. N. Li, "Some new dynamic inequalities on time scales," Journal of Mathematical Analysis and Applications, vol. 319, no. 2, pp. 802-814, 2006.

[17] M. Bohner and G. Sh. Guseinov, "The convolution on time scales," Abstract and Applied Analysis, Article ID 58373, 24 pages, 2007.

[18] C. J. Zhao and W. S. Cheung, "On opial-type inequalities with higher order partial derivatives," Applied Mathematics Letters, vol. 25, pp. 2156-2161, 2012. 


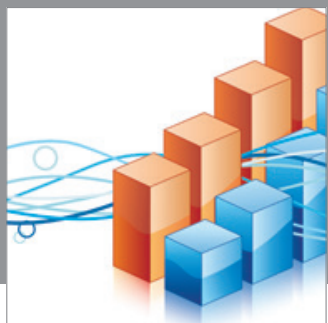

Advances in

Operations Research

mansans

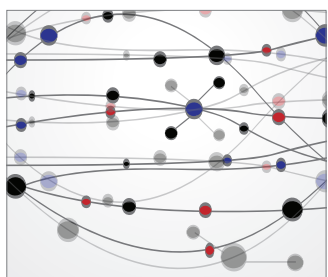

The Scientific World Journal
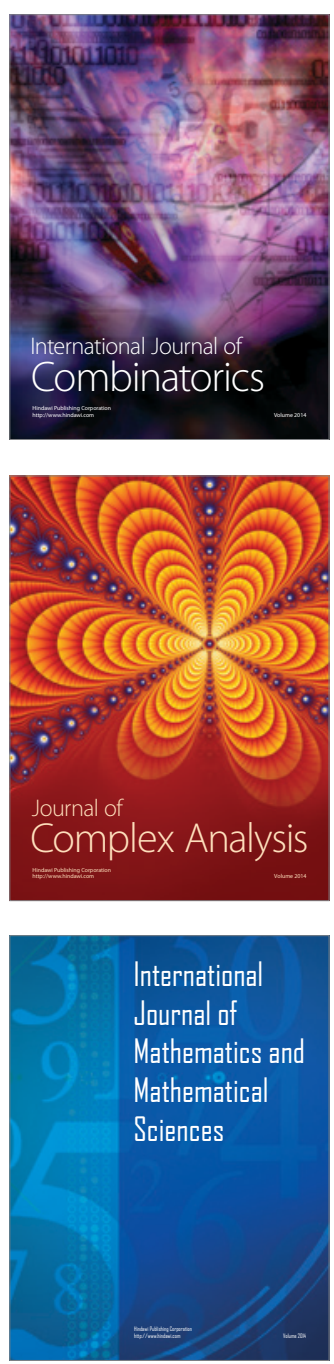
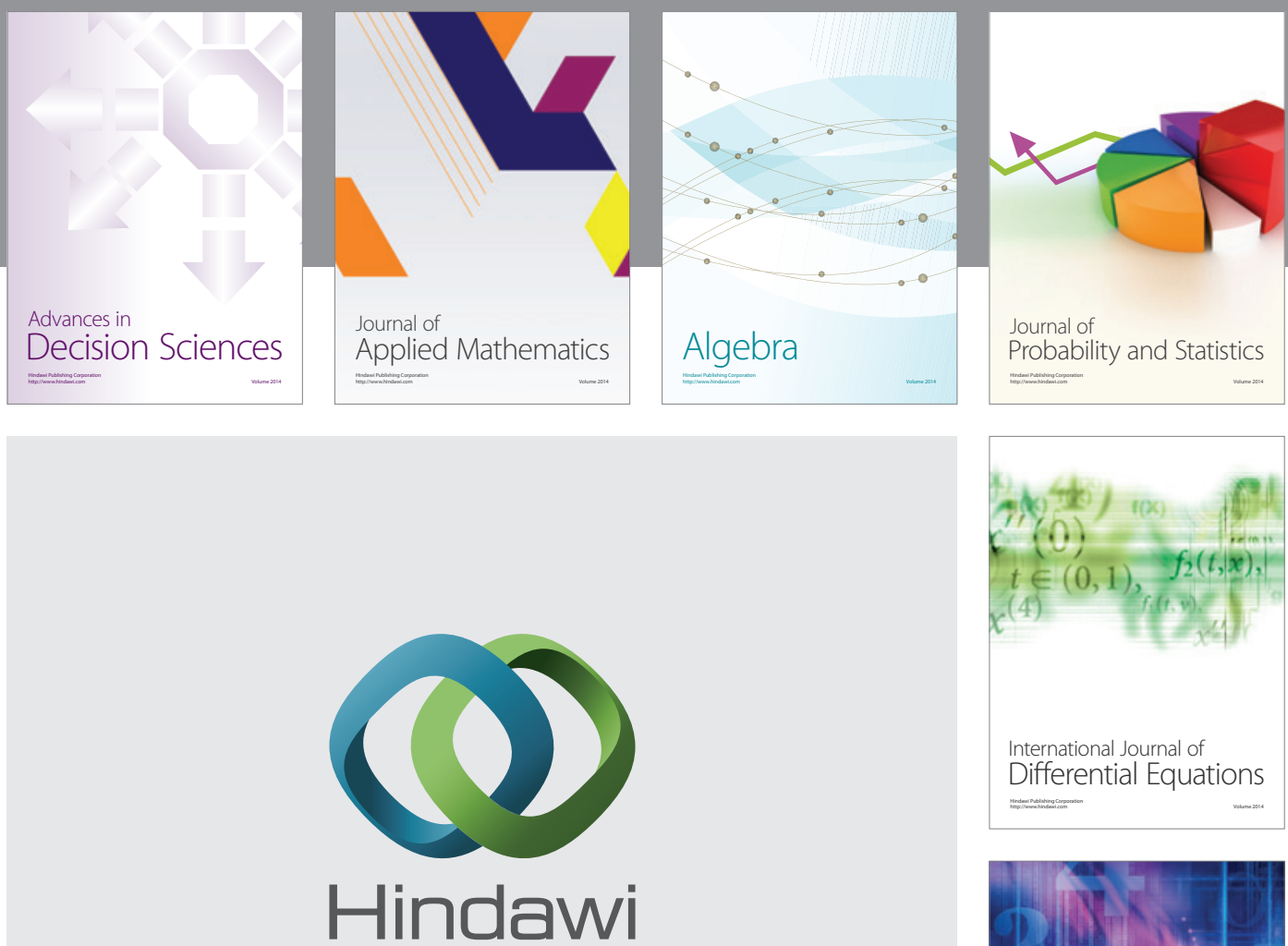

Submit your manuscripts at http://www.hindawi.com
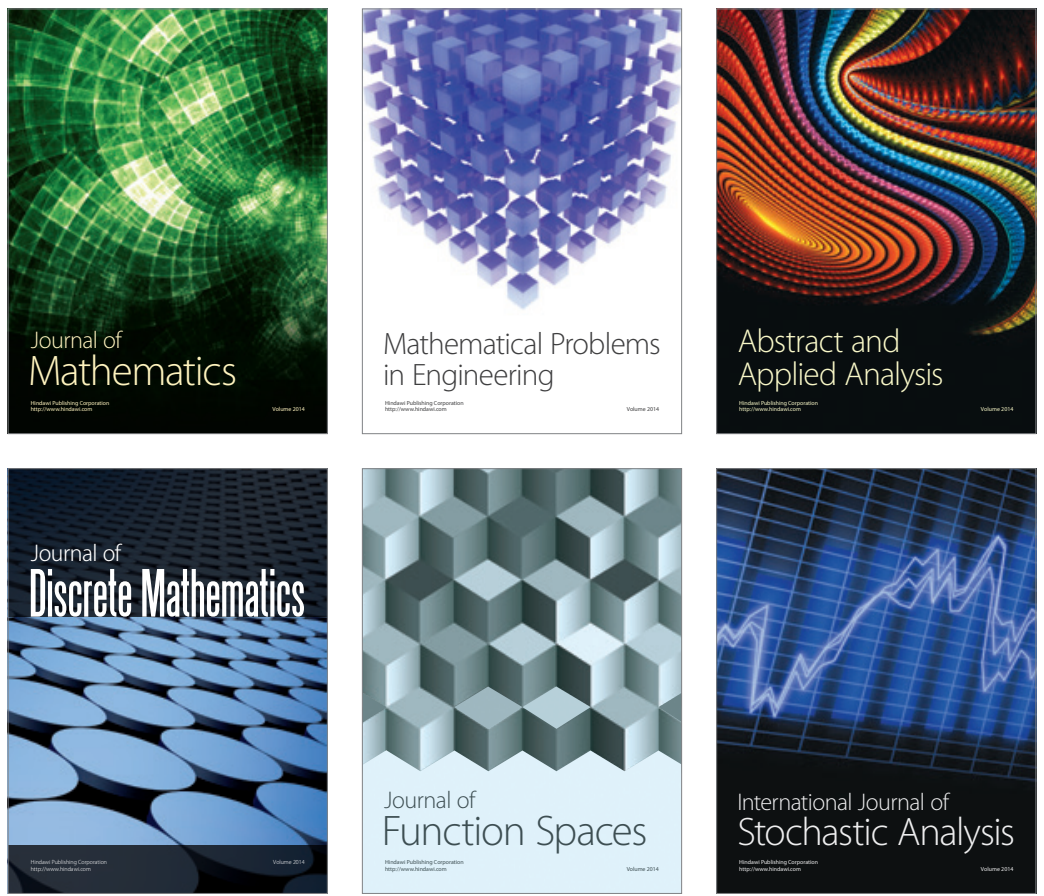

Journal of

Function Spaces

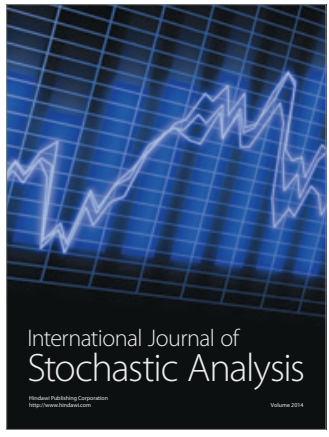

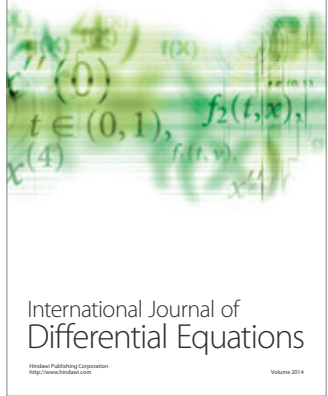
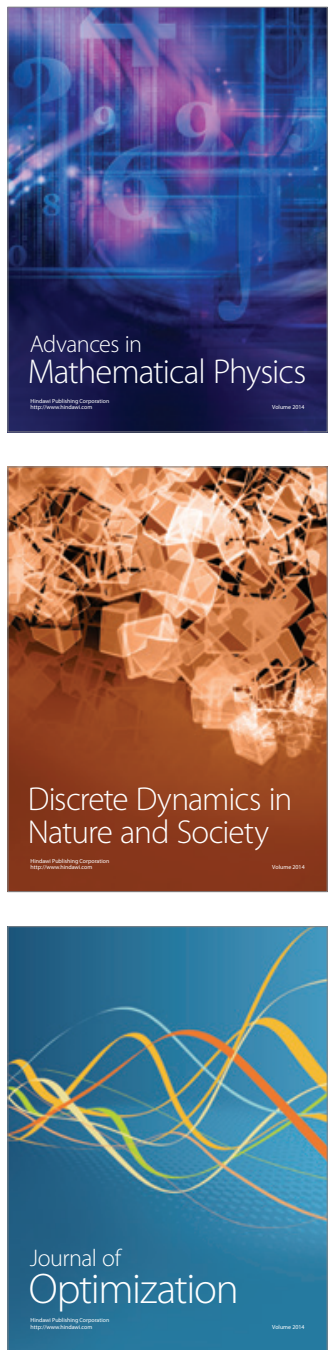